\title{
Toward a Computational Model of Social Relations for Artificial Companions
}

\author{
Florian Pecune \\ CNRS-LTCI; Telecom Paristech \\ Paris, France \\ florian.pecune@telecom-paristech.fr
}

\begin{abstract}
The objective of my Ph.D is to develop a computational model of social relations for artificial companions. Such a model should consider the representation of the social relation, its initialization and its dynamics over time. Moreover, the influence of this relation on the agent's decision-making should be modeled. In the proposed computational model described in this paper, the representation, initialization, and dynamics of the social relation are based on the research in Human and Social Sciences, considering the influence of individual and social parameters such as the personality of the agent or its social role.
\end{abstract}

Keywords-artificial companions; social dynamics; intimacy; decision-making;

\section{INTRODUCTION}

Machines nowadays own a prominent place in our daily lives. The study presented in [1] tends to show that the rules governing Human-machine social interactions are similar to those between two humans. Bledsoe's dream [2] to see computers befriend individuals is far from being a reality; however, many efforts are made to create virtual agents able to establish and maintain long-term relationships with their users. This is the particular case of Artificial Companions, following and assisting their "human masters" in everyday life. A companion can be defined as " $a$ robot or a virtual conversational agent that possesses a certain level of intelligence and autonomy as well as social skills that allow it to establish and maintain long-term relationships with users" [3]. One can cite for example the famous Tamagotchi, which, although it is relatively limited, owes its success to a concept very similar to the one implemented in virtual agents and robots developed nowadays.

The growing interest in artificial companions may be understood in the light of the works in Social Psychology. Indeed, there are many theories emphasizing the need for affiliation and its influence on human behavior [4]. Several works also highlight the impact of social relationships on the well-being of individuals [5]. If virtual companions are obviously not supposed to substitute human companions, they can still compensate the lack of social relations in particular situations. This can be, for example, the case for isolated seniors [6] in order to assist them in daily tasks, or simply to make small talks. Studies conducted by Turkle [7] also show that users are becoming more comfortable with the idea of interacting with these kinds of agents.

My Ph.D thesis is part of a national project ${ }^{1}$ aiming at developing virtual companions with different roles. To make each of them believable, they must be able to recall past interactions and their relationships have to change dynamically during the interaction [8]. The goal of my Ph.D is to give the capability to artificial companions to create relationships with users. For this purpose, a computational model of social relations should be defined to:

(1) determine the initial social relation a virtual companion should adopt toward the user depending on the context of the interaction (role of the agent, personality, etc.);

(2) compute how the social relation evolves through time during the interaction;

(3) model the influence of the companion's social relation on its decision making.

Indeed, the social relation of a companion should reflect the social context of the interaction. The companions' reactions should be different depending whether it interacts with a user to play a game or to teach a course of mathematics. These reactions should also reflect what happened during the interaction (for instance if the user abuses the companion). To model the companion's social relations, as a first step, we based our work on literature in Human and Social Sciences. Before introducing a first model of social relations, we present related works in the domain of virtual agents in the section below.

\section{RELATED WORKS}

\section{A. Artificial companions}

The number of projects featuring artificial companions has significantly increased over the past ten years. Started in 2004, the Cogniron project is one of the pioneers in this field [9]. The idea was to create a robot able to understand user's needs, and to assist him in various tasks. The robot must be able to perceive its environment, to analyze the various constraints inherent in achieving its task, and then to decide the best strategy to adopt. However, by focusing on the cognitive

\footnotetext{
${ }^{1}$ http://moca.imag.fr/
} 
aspect of the companion, the relational part has been missed. The robot is finally more an assistant than a real companion. The COMPANIONS project is more focused on the relationship between the user and the companion. The purpose is to encourage users to converse regularly with a virtual agent by telling him his day [10]. The model developed allows the agent to respond to emotional cues provided by the user, making the interaction more believable. The LIREC project focused on long-term interactions by modeling agent's memory and the evolution of dynamic relationships. The objective of this project was to develop a companion able to recall past interactions and adapt its behavior accordingly [11]. Beyond these research projects, there are many, more or less evolved, companions who have been or are still marketed today. In addition to the Tamagotchi mentioned above, there are robots like Pleo the dinosaur or Furby, responding to voice activation and learning behaviors over time. The virtual dogs Aibo and Genibo, as well as the virtual cat NeCoRo, try to mimic the behavior of the animals they represent. If $\mathrm{NeCoRo}$ is only able to purr and respond to caresses, Aibo and Genibo are able to move and detect obstacles. Finally, the autonomous robot PaPeRo offers many interesting features such as facial recognition and voice synthesis, making interactions with the user more enjoyable.

Although they are made for long-term relationships, few of these companions integrate an explicit social model for their relation with the user.

\section{B. Relational agents}

In the domain of virtual agents, some research has focused on relational agents, which aim at building relationships over several interactions with their users. However, they are mainly developed for a specific purpose and in a specific context. In [12] the authors propose an overview of the different areas in which relational agents could be used. Most of them are thus used in applications related to health and behavioral change, but one can also find them in leisure and domestic applications, or even in video games. Some relational agents have been developed yet. For instance, Laura [8] encourages users to exercise on a daily basis. Laura's behavior evolves during the interactions, through pre-scripted dialogues. The relationship is based on a stage model, which could be related to the notion of intimacy. Another example of relational agent is Rea [13], who adapts its dialog strategy according to the principle of trust. Representing a real estate agent, Rea uses small-talks to enhance the confidence of the user. Once the user becomes more confident with Rea, task-oriented dialog takes place. The agent Tinker [14] focuses on the recognition of users. Used as an information terminal in the Museum of Science in Boston, Tinker recognizes users it has already met using biometric identification (hand recognition). The agent can thus refer to previous interactions with the same user, providing customized advices. In Eva [15], the behavior is calculated according to its personality, mood, emotions and social relations toward the user. The relation is modeled according to two axes of dominance and appreciation, and evolves according to its emotions.

\section{Social Agents}

Unlike relational agents and artificial companions, social agents do not aim to model long-term relationships. Some of them, however, can stir up our interest due to the way they model the social relations and their dynamics. This is particularly the case of Avatar Arena, and the scenario in which a user must negotiate a schedule appointment with several agents [16]. Before each session, the value of appreciation between agents is fixed (low, medium, high), and it evolves according to the Congruity Theory described by Osgood and Tannenbaum [17]. This theory can also be found in FAtiMA [18], a computational model used in projects such as FearNot! or ORIENT. In Demeanour [19], relation also relies on the axis of appreciation and a second axis of dominance. The relationship evolves according to the personality of the agent, its mood, and the social attitudes expressed by the other party. This relationship may thus influence the non-verbal behavior of the agent, particularly in terms of posture and gaze. In SCREAM, emotions play an important role, changing the relationship according to their type, as explained in [20]. The representation of social relation in SCREAM is based on the same two axes already used in Demeanour: the social power represents the hierarchical relationship between two people and the social distance, the degree of appreciation. Prendinger adds to these two axes the notion of familiarity, also based on emotions, but evolving monotonically. Although it seems obvious that an agent's relationship toward a user will influence the agent behavior, there are few models considering the agent's belief of the supposed relationship of the user toward itself. PsychSim attempts to fill this gap by providing a framework in which the agent will take into account others' relations before planning their own actions [21]. Finally, Alpha Wolf gives us the opportunity to play a Cub [22], and interact with other wolves through howls, growls and whines. The dimension of dominance is the only one used here, and it just changes the animation of our cub depending on which wolf he is facing.

Although some computational models representing the impact of social relations on nonverbal behavior have been proposed, few of them take into account the dynamics of these relationships in the decision making process.

\section{THE REPRESENTATION OF VIRTUAL AGENTS'S SOCIAL RELATIONS}

Bickmore and Picard mentioned five different ways to model relationships in social psychology [8]. In dyadic models, two persons' behaviors are interdependent. A change in the state of one will thus produce a change in the other's state. Provision models are based upon one's expectations about what the other can provide to him. Economic models are rational models defining a relationship in term of costs and rewards. Dimensional models draw a relationship in a multidimensional space. Finally, stage models assume that the relationship can be represented by several distinct stages. 


\section{A. The Interpersonal Circumplex}

As shown in Section II, social relations can be represented by different dimensions (or axes). Although the most widely used dimensions refer to notions of dominance and liking, it is important to note that definitions of each of these dimensions vary depending on the model. In our model, we define liking as "a general and enduring positive or negative feeling about some person, object or issue" [23]. This notion is asymmetric [24] [20]-so not necessarily reciprocal- and is uncorrelated with notions of familiarity and solidarity [13]. However, in [19] as in [25], this notion is mixed with commitment. On dominance, there are also different interpretations depending on the model. In [19] and [20] dominance is defined as a hierarchical framework; in our work, we rely on [24] to define this dimension as "the capacity of one agent to affect the behavior of another". This influence is itself characterized by the resources and strategies available to the agent [26].

In order to model these two dimensions, we use the Interpersonal Circumplex [27], a model for conceptualizing and assessing interpersonal behaviors using two orthogonal axes: a vertical axis of dominance and a horizontal axis of liking, using the definitions given above (see Fig.1). There are many ways to map a social relation on this circumplex. Wiggins, for instance, defines a social relation by its angular coordinates, mapping the relation on the circle [27]. According to Leary, one can represent a relation using a labeled sub-part of the circumplex [28]. For instance, the subpart from $315^{\circ}$ to $360^{\circ}$ represents a "cooperative" agent, (high liking, low submission) while the sub-part from $270^{\circ}$ to $315^{\circ}$ describes a "docile" agent (low liking, high submission). But a social relation may also be drawn as a vector starting from the origin of the circumplex [29]. The two important features of this vector are its direction and its length. The direction, or angle, "suggests the predominant interpersonal theme" of the agent. A $45^{\circ}$ angle means that the agent shows an equal part of dominance and liking. An agent whose relational vector has a $60^{\circ}$ angle shows more dominance than liking. The vector length can be compared to interpersonal rigidity [30]. The longer an agent's vector is, the less it can adapt its behavior to a new situation.

In our model we use two representations of the circumplex, defining two types of relationships. For relations of the agent toward the user, we use the vector representation, in order to use Interpersonal Rigidity's rules. To model relations of the user toward the agent -which is a belief of the agent- we use the sub-part version (see Fig.1), as the agent can't be certain of the user's relation.
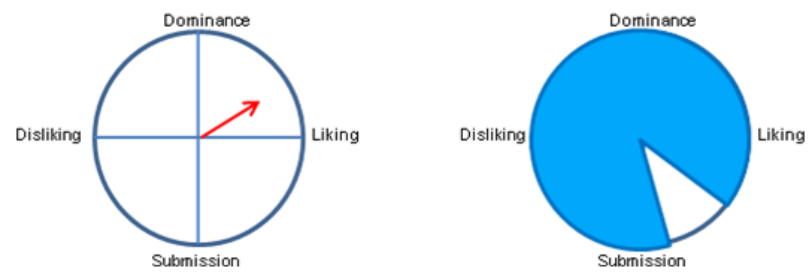

Fig. 1: Agent's relation toward user (left) and user's relation toward agent (right)

\section{B. Other dimensions}

Dominance and Liking are not sufficient to cover the whole domain of social relations. Svennevig thus set a list of dimensions related to this field [31]. Solidarity, for instance, is defined as "like-mindedness or having similar behavior dispositions" [13]. For instance, a French student doing an internship in Japan will feel a high solidarity upon other French students. As mentioned above, liking is sometimes mixed with commitment [19] [25], which represent the need to have a close relationship. Intimacy (or familiarity) describes the way in which the relationship evolves over time [13]. It can be defined in term of breadth (number of topics in common) and depth (public or private topics) as explained by the Social Penetration Theory [32]. Another important notion, especially in close relationships, is trust. In [13], trust is defined as a combination of affect (liking), familiarity and solidarity, therefore, it is not considered as a dimension.

In the case of artificial companions, we assume that the agent is committed and connected to the user. Therefore, we consider in our model the three following dimensions: dominance and liking (as a circumplex) and intimacy.

\section{SOCIAL RELATION INITIALIZATION}

In this section, we explore how to initialize the three dimensions considered in our model: liking, dominance, and intimacy.

\section{A. Intimacy}

The Social Penetration Theory [32] used in our model defines intimacy as a stage process. According to this theory, the relation can be drawn as a multilayered circle (see Fig.2), external layer representing superficial relation while the core represents the higher level of privacy. These layers have both breadth (number of common topics) and depth (private or public topics). As the relationship begins, the intimacy level is set as superficial.

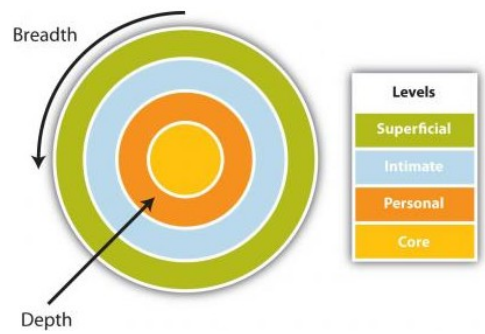

Fig.2 : Relationships layers according to Social Penetration Theory

\section{B. Social relations of the agent toward the user}

In [33], Scherer describes the interpersonal stance, saying they are "less shaped by spontaneous appraisal than by affect dispositions, interpersonal attitudes, and, most importantly, strategic intentions". In our model, we thus define the expressed relation as a combination of a strategic process

\footnotetext{
${ }^{2}$ http://educ5102.wikispaces.com/Social+Penetration+Theory
} 
(ideal relation) and a real feeling (felt relation). Therefore, we define agent's relation toward the user by three different vectors on the interpersonal circumplex, each of them initialized by different processes.

- The felt relation refers to the global feeling of the agent (e.g. the agent doesn't like the user). It evolves according to the interaction with the user. For instance, if the user insults the agent several times, or shows him disrespect, the latter will surely like the user less. Agent's personality plays a great role here, as it initializes the felt relation during the first encounter with someone [34].

- The ideal relation indicates the relation the agent would like to express in a particular situation (e.g. a teacher would like to show high dominance to his students for his first class). Length and direction of the vector strongly depends on the role played by the agent. An agent playing a stubborn teacher will have a long dominant vector whereas a diplomatic one will have a shorter vector.

- The expressed relation therefore represents the relation the agent expresses at a given moment. It can be calculated by the combination between the felt and ideal relations described above. As stated in [19], mood has an impact on the liking value (e.g. an agent in a bad mood will be more hostile). The status of resources held by the agent influences the dominance value [26].

\section{Social relations of the user toward the agent}

As showed in [21], "a key factor in human social interaction is our beliefs about others". Therefore, in our model, we propose a representation of the user's relation toward the agent.

- The estimated relation shows the relation that the agent thinks the user feels toward it (e.g. the agent thinks that the user likes him). This relation comes out the Theory of Mind [35] and can be different than the relation felt by the user.

- The desired relation is the relation that the agent wants that the user expresses (e.g. the agent wants the user to be submissive). It comes out from the ideal relation described above, according to Interpersonal Complementarity [36] which is basically a symmetry of the ideal relation upon the appreciation axis. If the agent would like to express both dominance and liking, then it will expect the user to express submission and liking.

- The perceived relation refers to the relation perceived by the agent at a given time $t$. It depends on the personality and the culture of the agent [37]. Perceived relation can be different than the relation expressed by the user and can also be different than the estimated relation: the agent may think that the user is usually submissive toward itself, but perceive clues of dominance during a particular interaction. The perceived relation also influence the estimated relation. If the agent perceives repeated cues of dominance from the user over time, it will update its beliefs consequently.

All the relations and parameters influencing them are illustrated in Fig.3. These relations will then be used to define the agent's behavior and its strategies.

\section{SOCIAL RELATION DYNAMICS}

Once we have defined and initialized agent's social relations, we need to know how they evolve during interactions with the user.

\section{A. Intimacy evolution}

Intimacy dynamics are based on self-disclosure. As depicted in [32], the more time an agent spends with the user, the more likely both of them are to self-disclose intimate thoughts as the layers shed away. In [20], authors use a factor to determine how rapid the agent gets familiar with the user. For instance, there is a strong correlation between the liking value and self-disclosure [38], meaning that an agent will increase its intimacy faster if it likes the user. Moreover, agents engaged in intimate disclosures tend to be liked more than others disclosing at lower levels. In [39], the authors propose a model of disclosure decision-making during a single interaction. This model takes into account background factors like culture and personality, beliefs like relationship quality and also the outcomes of this disclosure, for a dynamic evolution. Our model implements the multilayered circle described in Fig.2 according to the following rule : the more the agent learns about the user (breadth improvement), the higher its intimacy value becomes (depth enhancement).

\section{B. Dominance / Liking dynamics}

We saw in related works that emotions can influence social relations. In [40], authors draw up a list of emotions and modeled their impact on dominance and liking dimensions, based on literature. A comparable work has been done in [20] and [15]. For example, an agent feeling a pride emotion elicited by the user will feel more dominant toward him.

However, as a first step, we rely on the Congruity Theory [17] as it appears in [16] to model the relational dynamics between the user and the agent. We then use a triangular schema between an agent $\mathrm{A}$, a user $\mathrm{U}$ and a situation $\mathrm{S}$. It describes A's relation toward the user $\mathrm{U}$ (felt relation), A's relation toward a situation $\mathrm{S}$ (ideal relation) and A's assumption about $\mathrm{U}$ 's relation toward $\mathrm{S}$ (desired relation). For instance, the agent likes the user, wants to show dominance and thus awaits submission from U. We consider that the interaction is congruent when U's assertion about $\mathrm{S}$ (perceived relation) is consistent with the desired relation (e.g. the user shows submission). In that case, the felt relation is modified (attracted) by the ideal relation weighted by intimacy value: the more the agent is familiar with the user, the less its felt relation will be modified. However, we consider incongruity when U's assertion about $\mathrm{S}$ is different than the one expected. Considering the same example, there is incongruity when the user shows dominance. To fix this incongruity, the agent may try different strategies: (a) change its felt relation toward the user, (b) change its own ideal relation or (c) try to change user's expressed relation. Dominance and liking dynamics will thus depend on the strategy of the agent. 


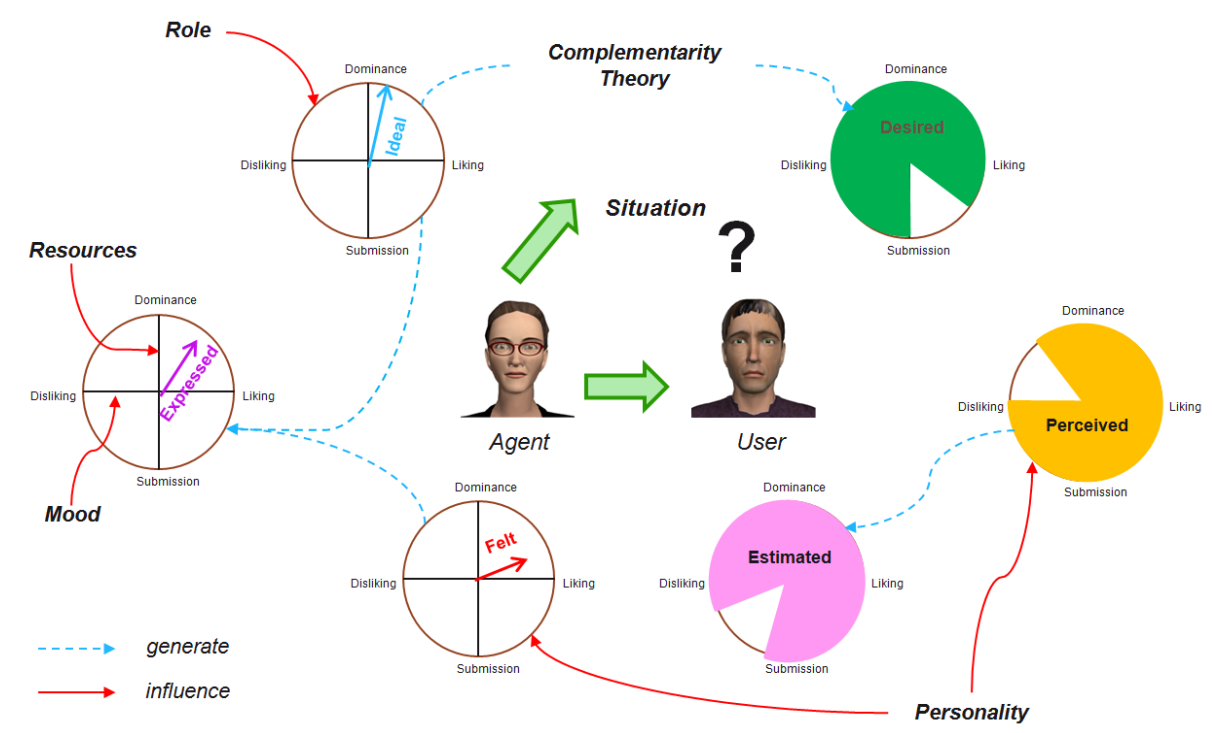

Fig.3: Dynamics of the agent's social relations and their influences, in the light of Congruity Theory.

\section{CONCLUSION AND FUTURE WORKS}

In this paper, we presented a model of social relations for an artificial companion. These relations are defined by three dimensions: dominance, liking and intimacy. The latter is based on Social Penetration Theory, and is represented by a multilayered circle going from superficial to core relationship. Dominance and liking form the two axes of an Interpersonal Circumplex. The agent's relation toward the user is drawn by a vector, while the user's relation toward the agent is a subpart of this circumplex. Using the circumplex, we defined six different kinds of relations (felt, ideal, expressed, estimated, desired and perceived), each of them influenced by social and individual parameters. Dynamics of those six relations rely on Congruity Theory: if the agent's perceived relation isn't consistent with the desired relation, the agent will thus have to adopt a strategy consequently.

Those strategies are part of our future works. Indeed, we still need to model how social relations influence decisions made by an agent. In [26], Raven draw up a list of dominant strategies based upon predicted costs and rewards, as well as upon the agent's goals. In [41], authors stated that one can be easily influenced by someone you like, knowing that this influence is less important if the final decision has a high personal importance [8]. Instead of using dominant strategies, the agent may thus try to befriend the user to influence him more efficiently.

Our next step is to implement the computational model depicted in the previous sections. For instance, we need to model how exactly the role of the agent influences its ideal relation. Should we use a probabilistic model, fuzzy logic, or should we simply fix dominance and liking values according to this role? We also have to keep in mind how to evaluate our model, and which part we want to evaluate : should we assess our companion's performances regarding its role or measure user's satisfaction?

\section{ACKNOWLEDGMENT}

This research has been supported by the ANR Project MoCA

\section{REFERENCES}

[1] B. Reeves and C. Nass, The Media Equation: How People Treat Computers, Television, and New Media Like Real People and Places, Cambridge University Press, 1996.

[2] W. Bledsoe, "I Had a Dream: AAAI Presidential Address, 19 August 1985," AI Magazine vol.7, $n^{\circ} 1$, pp. 57-61, 1986.

[3] M. Lim, "Memory Models for Intelligent Social Companions," Human-Computer Interaction: The Agency Perspective Studies in Computational Intelligence vol.396, pp. 241-262, 2012.

[4] A. Maslow, "A theory of human motivation.," Psychological Review vol.50, $n^{\circ} 4$, pp. 370-396, 1943.

[5] E. Berscheid and L. Peplau, "The Emerging Science of Relationships," in Close Relationships, New York, W.H. Freeman, 1983, pp. 1-19.

[6] S. Kriglstein and G. Wallner, "HOMIE: An Artificial Companion for Elderly People," in CHI'05, Portland, 2005.

[7] S. Turkle, "In Good Company: On the treshold of robotic companions," in Close Engagements with Artificial Companions: Key social, psychological, ethical and design issues, John Benjamins, 2010, pp. 1-10.

[8] T. Bickmore and R. Picard, "Establishing and maintaining longterm human-computer relationships," ACM Transactions on Computer-Human Interaction (TOCHI) vol.12, $n^{\circ} 2$, pp. 293327, 2005.

[9] A. Clodic, R. Alami, V. Montreuil, S. L., B. Wrede and A. Swadzba, "A study of interaction between dialog and decision for human-robot collaborative task achievement," in $R O$ $M A N^{\prime} 07,2007$.

[10] M. Cavazza, S. d. 1. C. R. and M. Turunen, "How Was Your Day? a Companion ECA,," in $A A M A S^{\prime} 10$, Toronto, Canada, 
2010.

[11] P. Gomes, A. Paiva and C. Martinho, "Episodic memory retrieval through re-appraisal of past events," in IVA'10, Philadelphia, 2010.

[12] R. Campbell, M. Grimshaw and G. Green, "Relational Agents: A Critical Review," The Open Virtual Reality Journal, vol.1, pp. 1-7, 2009.

[13] T. Bickmore and J. Cassell, "Social dialogue with embodied conversational agents," in Advances in natural multimodal dialogue systems vol.30, 2005, pp. 23-54.

[14] D. Schulman, M. Sharma and T. Bickmore, "The Identification of Users by Relational Agents," in $A A M A S^{\prime} 08$, Estoril, Portugal, 2008 .

[15] Z. Kasap, M. Ben Moussa, P. Chaudhuri and N. MagnenatThalmann, "Making Them Remember-Emotional Virtual Characters with Memory," Computer Graphics and Applications, IEEE vol.29, $n^{\circ} 2$, pp. 20-29, 2009.

[16] T. Rist, M. Schmitt, C. Pelachaud and M. Bilvi, "Towards a Simulation of Conversation with Expresive Embodied Speakers and Listeners," in $C A S A^{\prime} 03,2003$.

[17] C. Osgood and P. Tannenbaum, "The Principle of Congruity in the Prediction of Attitude Change," Psychological Review vol.62, $n^{\circ} 1$, pp. $42-55,1955$.

[18] M. Y. Lim, J. Dias, R. Aylett and A. Paiva, "Creating Adaptive Affective Autonomous NPCs," Autonomous Agents and MultiAgent Systems vol.24 $n^{\circ} 2$, pp. 287-311, 2012

[19] D. Ballin, M. Gillies and I. Crabtree, "A Framework For Interpersonal Attitude And Non-Verbal Communication In Improvisational Visual Media Production," in First European Conference on Visual Media Production IEE, London, UK, 2004.

[20] H. Prendinger, S. Descamps and M. Ishizuka, "Scripting affective communication with life-like characters in web-based interaction systems," Applied Artificial Intelligence: An International Journal, vol.16, $n^{\circ} 7-8$, pp. 519-553, 2002

[21] S. Marsella, D. Pynadath and S. Read, "PsychSim: Agent-based modeling of social interactions and influence," in $I C C M^{\prime} 04$, 2004

[22] B. Tomlinson and B. Blumberg, "Social Synthetic Agents," Computer Graphics vol.26, $n^{\circ} 2,2002$.

[23] L. Moshkina and R. Arkin, "On TAMEing robots," in IEEE International Converence on Systems, Man and Cybernetics, 2003.

[24] R. Prada and A. Paiva, "Social Intelligence in Virtual Groups," Studies in Computational Intelligence vol.140, pp. 113-132, 2008

[25] K. Isbister, Better Game Characters by Design, A Psychological Approach, Morgan Kaufmann, 2006.

[26] B. Raven, "The Bases of Power and the Power/Interaction Model of Interpersonal Influence," Analyses of Social Issues and Public Policy vol.8, $n^{\circ}$, pp. 1-22, 2008.
[27] J. Wiggins, P. Trapnell and N. Phillips, "Psychometric and geometric characteristics of the Revised Interpersonal Adjective Scales (IAS-R)," Multivariate Behavioral Research vol.23, $n^{\circ} 4$, pp. 517-530, 1988.

[28] T. Leary, Interpersonal diagnosis of personality: A functional theory and methodology for personality evaluation, New York: Ronald Press, 1957.

[29] M. Gurtman, "Circular reasoning about circular assessment," in Handbook of Interpersonal Psychology: Theory, Research, Assessment, and Therapeutic Interventions, Horowitz, L.M ; Strack, S., 2011, pp. 299-311.

[30] T. Tracey, "Interpersonal rigidity and complementarity," Journal of Research in Personality vol.39, $n^{\circ} 6$, pp. 592-614, 2005.

[31] J. Svennevig, Getting Acquainted in Conversation, Philadelphia: John Benjamins, 1999.

[32] I. Altman and D. Taylor, Social Penetration: The Development of Interpersonal Relationships, New York, 1973.

[33] K. Scherer, "What are emotions? And how can they be measured?," Social Science Information vol.44, $n^{\circ} 4$, p. 695-729, 2005.

[34] P. Trapnell and J. Wiggins, "Extension of the Interpersonal Adjective Scales to Include the Big Five Dimensions of Personality," Journal of Personality and Social Psychology vol.59, $n^{\circ} 4$, pp. 781-790, 1990.

[35] S. Baron-Cohen, A. Leslie and U. Frith, "Does the Autistic Child Have a "Theory of Mind" ?," Cognition, vol. 21, $n^{\circ} 1$, pp. 37-46, Octobre 1985

[36] P. Sadler, N. Ethier and E. Woody, "Interpersonal Complementarity," in Handbook of interpersonal psychology: Theory, research, assessment, and therapeutic interventions, New York, Horowitz, L.M. ; Strack, S., 2011, pp. 123-142.

[37] C. Clavel, A. Rilliard, T. Shochi and J.-C. Martin, "Personality differences in the multimodal perception and expression of cultural attitudes and emotions," in $A C I I^{\prime} 09$, Amsterdam, Netherlands, 2009.

[38] N. Collins and L. Miller, "Self-disclosure and liking: a metaanalytic review.," Psychological Bulletin vol.116, $n^{\circ} 3$, pp. 457475,1994

[39] K. Greene, V. L. Derlega and A. Mathews, " Self-disclosure in personal relationships," in Cambridge handbook of personal relationships, Cambridge, UK, Cambridge University Press, 2006, pp. 1268-1328.

[40] M. Ochs, N. Sabouret and V. Corruble, "Simulation of the dynamics of Non-Player Characters' emotions and social relations in games," IEEE Transactions on Computational Intelligence and AI in Games vol.1, n²4, pp. 281-297, 2009.

[41] D. Schulman and T. Bickmore, "Persuading users through counseling dialogue with a conversational agent," in PERSUASIVE'09, Claremont, USA, 2009. 